\title{
The Impact of Health Education or Health of the Victims of the Earthquake in Disaster Area
}

\author{
Wei Liu \\ Party School of the Jiangsu Provincial Committee of CPC \\ School of International Relations and Public Affairs, Fudan \\ University
}

\author{
Lanping Jiang \\ Peking Union Medical College \\ lanpjiang2000@126.com
}

\author{
Benhui Shi \\ Sichuan Academy of Social Science \\ scassshibenhui@126.com \\ (*corresponding author)
}

\author{
Xiaolin Tangs \\ China Medical University \\ medicaltxl@163.com
}

\begin{abstract}
Earthquakes, floods, mudslides, wars and other major traumatic events can have on people's physical and mental health caused serious damage, leading to muscle tension, loss of appetite, mental panic, mood disorders, behavioral disorders and other stress reactions, and young people are in the physical and psychological rapid growth, physical and mental health are more susceptible to traumatic events. Earthquakes are a common type of devastating natural disasters, crises, with sudden, social urgency, high uncertainty and the impact [1] is a serious incident (critical incident). As Solomon said, the critical incident refers to a cause that cannot resist the feeling of self-generated and lost control of the situation. Individuals in such stress situations, often find it difficult to solve, it is difficult to grasp. The disruption of normal life and inner tension continue to savings followed by a loss even thinking and behavior disorders, and enter into a state of imbalance, that is in a state of psychological crisis. According to the theory of stress, personal reactions to stress are not only the nature of the sources of stress, but also the state of stress modifiers (stress moderators) is related. Stress modifiers include factors of personality, coping style, social support and external resources, and regulate the experience of stress and stresseffects individuals. Faced with such a severe earthquake emergencies, victims have their own coping strategies that affect the victims of the earthquake's psychological reaction, thereby determining the psychological crisis intervention in key areas and populations.
\end{abstract} Health

Keywords- Health Education; Earthquake Disaster; Victims

\section{INTRODUCTION}

Subjected to severe psychological trauma stricken, adolescent will have psychosomatic disorders and incidence is significantly higher than the non-affected areas of the same age teenagers. Girls' stricken extent of the damage is more serious physical and mental health than boys. It is consistent with previous studies [11, 13]. High age youth psychosomatic disorders was higher than the low age, it may be associated with high youth ages traumatic event, itself concerns negative perception lower than age-related.
Stricken adolescent with physical symptoms of psychosomatic have disorders of major headache, stomach upset, loss of appetite, difficulty breathing, palpitation, fatigue, muscle pain, psychological symptoms of insomnia, nightmares, easily frightened, tension, depression, etc. Age, mental endurance capacity are a major factor in psychosomatic disorders psychological impact score, and sleep state, age, performance status score the greater impact on the body. From Etiology analysis, physical and mental health are by bio - psycho - social and other factors affect [15] physical symptoms and psychological symptoms related to both each other and interact. This verifies the stricken youth psychosomatic disorder is the result of internal and external interaction, live, work and major psychological trauma and other stress effects on susceptible individuals, and it can induce psychosomatic disorders. Adolescent psychosomatic disorders in disaster areas give timely and effective psychological interventions; they can get rid of the psychological crisis as soon as possible to re-adapt to life. Wenchuan earthquake disaster area for physical and mental health of young people caused serious damage.

\section{A FIRE HAZARD AND THE RISK OF EARTHQUAKE} DISASTER VICTIMS IN TEMPORARY RESETTLEMENT SITES

Personnel are highly intensive, on-site chaos. Department of victims in such places as "shock absorbers" for the common purpose is gathering together to form spontaneously, and it is recognized by everyone in subconscious open land with a certain security in urban areas, squares, stadiums and other large-scale has become the victims of choice. Thus, within a city all displaced people will spontaneously gathered in the few square halls or stadium, a highly dense, chaotic place. 1.2 with spacing small fire, fire load is "hidden point", the fire spread quickly. Such establishments affected people spontaneously formed Department, or "make the best" use of flammable plastic sheeting, color of the cloth, nylon, canvas and other materials hastily erected, or they use tents provided by the local civil affairs department building. 
In short, the anarchy makes these "shock shed" no unified planning in the distribution: the rope is used to secure the tent with the addition of the "veneer style", "back to back" type, the minimum spacing between the tent fire ; clothing and bedding and other items brought out when victims escape flooded the inside, making the fire load suddenly increase. Taking these factors, in the event of fire, the fire spreads rapidly, forming serious situation.

With frequent use of fire, due to earthquake damage, is often accompanied by power supply facilities, then the use of candles and other open flames lighting has become a necessity, smoking, mosquito repellent and other acts will also greatly increase the frequency of the use of fire.

Electrical lines are with chaos and load; electrical wiring fires occur to incentives more. If there is the power supply equipment, electrical wiring random access phenomenon will be inevitable. Part of the affected people do not consciously use electrical power in the "shock shed", which secretly increase the capacity in the electrical wiring, and electrical equipment. The metal odds are often unable to set up a reliable ground protection that is not so easily to cause electrical short circuit, plus lighting surface temperature insulated parts that do not fire when near combustible materials and other reasons, such places have larger fires of an electrical circuit.

There are no fire equipment configuration and no fire channel. As the "beginning of construction" phase of the "anarchy" state makes these "shock shed" in the distribution appear disorganized, but they generally have no place in the fire equipment configuration, traffic-free roads tend to be "shock shed". A fire victims has low self-help capabilities, when the fire broke out, fire fighting is to enter effective that is a worrying problem.

Staffs are complicated; it is difficult for evacuation escape, which could easily lead to heavy casualties. Among the dense crowd and wounded, there are the elderly, women, children and other vulnerable groups, in case of fire, the ability of such persons to escape with low self-help, plus a fire is often accompanied by a large number of $\mathrm{CO}$ and other toxic fumes that generated most likely cause even more significant than the earthquake disasters of casualties.

The extent of the trauma of the earthquake is continuing to affect the health of residents. Studies have shown that the nature of the disaster itself can stimulate considerable extent that determines the individual's physical and mental reaction conditions. These risk factors, such as between the objective and subjective psychological adaptation and subsequent injury are to face his body, relatives and friends of the disabled, missing or death, loss of housing and property, etc.. They are often making them feel very helpless, and fear, and the existence of "dose - effect" (dose-effect) relationship, life-threatening and property damage caused by the disaster that is the more serious, the worse hit by the disaster stress symptoms and physical and mental condition. This study also shows that the seismic moment of trauma experience still causes long-term effects on the health of residents, the shadow of the earthquake is still not dispersed, the trauma of the earthquake, the population are exposed to high psychological intervention that is an ongoing process, long- term efforts needed. On the other hand, the influence of the earthquake trauma at this stage is not as factors such as social support post-disaster situation. Studies have shown that with the passage of time after the disaster, the immediate impact of the disaster factors gradually weakened after a disaster affecting factors on post-traumatic stress reactions gradually increased. Social support on the one hand is to the individual under stress increase protection, corresponding buffer provoked, on the one hand is to maintain general good emotional experience that plays an important role. The affected community residents concerned, which is made with the help of psychologists that are very positive.

Whether in urban or rural areas, whether with economic development, for everyone concerned, health and education are the two cornerstones of its capital, which has a very complex interactions between human society is the basis for the development of people's health and education. The impact of low health literacy is higher than the impact of education on health, income, unemployment and race and other factors. For residents of the affected areas, the continuous improvement of health literacy is the most urgent needs. Therefore, in the affected areas, we should carry out health education and health promotion, you need to fully exploit the various social resources, continue to strengthen all kinds of people on social behavior intervention, gradually increase the knowledge and awareness of health-related and health-related behaviors formation rate.

\section{THROUGH THE SYSTEM, TO CARRY OUT A SERIES OF HEALTH EDUCATION ACTIVITIES CAN IMPROVE RESIDENTS' HEALTH KNOWLEDGE AND AWARENESS}

It also shows that only by understanding the needs of the masses, using different means of communication, targeted to attract the masses, in order to truly mobilize the enthusiasm of the masses to actively participate in activities to improve the effect of the activities to achieve the purpose of health education. With the changing social and economic development as well as dietary habits of the population, the incidence of chronic diseases and increasing the proportion of deaths are caused by increasingly heavy economic burden. Therefore, prevention of chronic non-communicable diseases has become a focus of community health work. There is no doubt the system residents carry out health education activities in a cost-effective activities. The survey found that residents of unhealthy dietary habits, lifestyle, and habits are risk factors for chronic non-communicable diseases, and health education is in favor of risk factors good management, it can correct concept that people rely on drugs to treat chronic diseases, "health education needs the residents' health education-oriented measures with the effect of more general way. In developed regions, such as Shenzhen, in fact, everyone has some knowledge. If there is no demand investigation, we cannot accurately grasp the needs of the residents of the health knowledge. Health education in the normal way would result in duplication of mission of knowledge, and its the effect will be lower. "Health education needs the residents' health education measures oriented effect in some high cultural quality of the community that is better than the low cultural quality of the 
community, the traditional material for propagation material plane is typical for rural residents word, readability is poor, poor publicity. In recent years, China's rural community health education remains the traditional health education, such as publicity windows, publicity boards, consulting, lectures and other forms of mainly older content, broad, unattractive and guidance. With the development of information technology and leap, multimedia communication materials for the development of production have become the mainstream. So in the process of health communication, it should be based on different target groups for age, sex, education level, ethnicity and other factors, actual demand, targeted development and production of digital multimedia communication materials, in order to carry out health education in different populations and health promotion.

\section{AFTER AN EARTHQUAKE, THE RISK OF INFECTIOUS DISEASES OCCUR TO STRICKEN RESIDENTS, COMMON COLDS INCREASES}

On the one hand, the occurrence of these diseases can be achieved through health awareness and health knowledge. If we improve behavior, it will be reduced, even after illness, these diseases can also improve the behavior and health seeking behavior and eased or eliminated. On the other hand, health education can improve health awareness and health knowledge; improve behavior and health seeking behavior. Therefore, the role of health education on how to improve the health status of residents in the quake-hit areas is necessary. Study: Health education in post-disaster recovery, in addition to continue use of oral preaching and writing, but also according to the prevailing epidemic dynamics supplement produced new promotional material, the use of windows, banners, home-face advocacy, intensive training, peer comprehensive education and other means. Through health education, it advocates healthy health behaviors in order to improve people's awareness and self-care skills. Based on this, the project spread through the home, lectures, peer education, banners, paid in the form of promotional materials, special events, etc., to the disaster area residents disseminate educational knowledge. The evaluation results show: health education intervention, the three provinces surveyed residents in the overall level of health knowledge were significantly improved after the intervention level of knowledge and awareness of each basic more than $80 \%$, indicating that residents have a better grasp of core health information. From another aspect, but also reflects the spread of the main face to face various forms of intervention that has achieved certain results to achieve the desired purpose of the intervention.

\section{HEALTH EDUCATION, HEALTH KNOWLEDGE AS AN IMPORTANT PART OF PROMOTION}

The health education and health knowledge are an important promotion content, through the issuance of a variety of health and epidemic prevention and post promotional materials, lectures, radio and other forms of knowledge to improve the health and epidemic prevention residents in the effective prevention of the occurrence of post-infectious diseases played an important role. Health education coverage residents reached $89 \cdot 77 \%$, health and epidemic prevention knowledge and awareness reached 92 • $72 \%$, a description of these forms of health education in the affected areas is feasible and can prevent the occurrence of infectious diseases as a reference for all kinds of natural disasters in the future. Knowledge of rural residents and migrant population health and disease prevention awareness is low, suggesting that in the future, the earthquake relief work should strengthen the rural and migrant population health and disease prevention of knowledge propaganda, all the residents are to understand and master the health and epidemic prevention knowledge. Health education in the future, you can focus on the members of the various settlements vaccination teams were deep training, so they organized residents' hygienic knowledge training.

\section{CONCLUSIONS}

Through health education, health knowledge among residents of earthquake-stricken areas they have improved health behavior. Initial formation of "catch-face focus groups propagate, that spreads material payment security coverage, local special events to stimulate active participation" of the health education intervention model that should be implemented in the earthquake area, in order to continue to promote the health of the residents of the affected areas.

\section{REFERENCE}

[1] Liu Hong. Analysis earthquake disaster victims in coping strategies condition [J] Chinese Journal of Health Psychology, 2010,03: 310313.

[2] Huang Yong. Earthquake disaster victims in fire prevention strategy in place a transition point [J] Fire Science and Technology, 2013,09: 1042-1044

[3] Jie Xinghong. Solution Preliminary earthquake disaster victims in temporary resettlement sites of fire safety management measures [J] managers, 2009, 12: 105.

[4] Lu Yiming. Gansu earthquake disaster stricken areas of health promotion and health education intervention study [D]. Lanzhou University, 2013.

[5] Chen Yulan, Gong Bo. Wave earthquake-stricken areas of a hospital medical staff to deal with mental health and stress survey [J] Chinese Rural Health Service Administration, 2014,09: 1119-1121. 